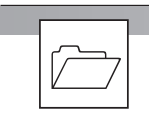

Endokrynologia Polska

DOI: 10.5603/EP.a2020.0003

Volume/Tom 71; Number/Numer 2/2020 ISSN 0423-104X

\title{
Medullary thyroid carcinoma of unknown primary origin with synchronous finding of papillary thyroid carcinoma
}

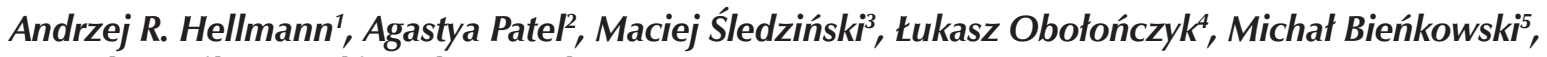 \\ Bogusław Mikaszewski ${ }^{6}$, Łukasz Kaska ${ }^{1}$ \\ ${ }^{1}$ Department of General, Endocrine, and Transplant Surgery, Medical University of Gdańsk, Gdańsk, Poland \\ ${ }^{2}$ Scientific Circle of Surgery, Department of General, Endocrine, and Transplant Surgery, Medical University of Gdańsk, Gdańsk, \\ Poland \\ ${ }^{3}$ Department of Emergency Medicine, Medical University of Gdańsk, Gdańsk, Poland \\ ${ }^{4}$ Department of Endocrinology and Internal Medicine, Medical University of Gdańsk, Gdańsk, Poland \\ ${ }^{5}$ Department of Pathology, Medical University of Gdańsk, Gdańsk, Poland \\ ${ }^{6}$ Department of Otolaryngology, Medical University of Gdańsk, Gdańsk, Poland
}

Key words: medullary thyroid carcinoma; papillary thyroid carcinoma

A 34-year-old male presented to the Otorhinolaryngology Outpatient Clinic with primary complaint of non-specific neck pain and was found to have left-sided submandibular lymphadenopathy on physical examination. A fine needle aspiration biopsy (FNAB) of the lymph nodes (LN) was performed. An initial diagnosis of malignant salivary gland neoplasm was made. To further delineate the tumour location, magnetic resonance imaging (MRI) of the neck was done. It revealed pathologically enlarged LN; however, no abnormalities in the salivary glands were noticed (Fig. 1). Therefore, PET scan with ${ }^{18} \mathrm{~F}$-FDG was carried out to locate the primary tumour site. It revealed an active metabolic process

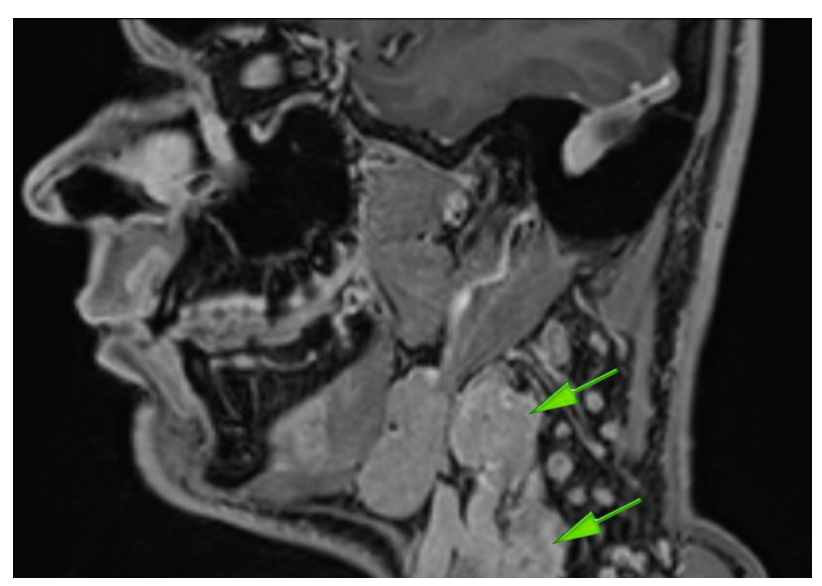

Figure 1. MRI with pathological lymph nodes with no abnormalities in salivary glands (green arrows) in several LNs of the upper and middle neck regions (Fig. 2). In light of these imaging studies, the patient was taken for bilateral tonsillectomy, adenoidectomy, and selective neck dissection of level II LNs. Histopathological examination revealed macro-metastasis of medullary thyroid carcinoma (MTC) in these lymphoid tissues. On immunohistochemistry, the specimens stained positively for synaptophysin, chromogranin A, and calcitonin. Meanwhile, the serum calcitonin level was $300 \mathrm{ng} / \mathrm{mL}$.

With the suspicion of thyroid cancer, a multidisciplinary discussion was held, which qualified the

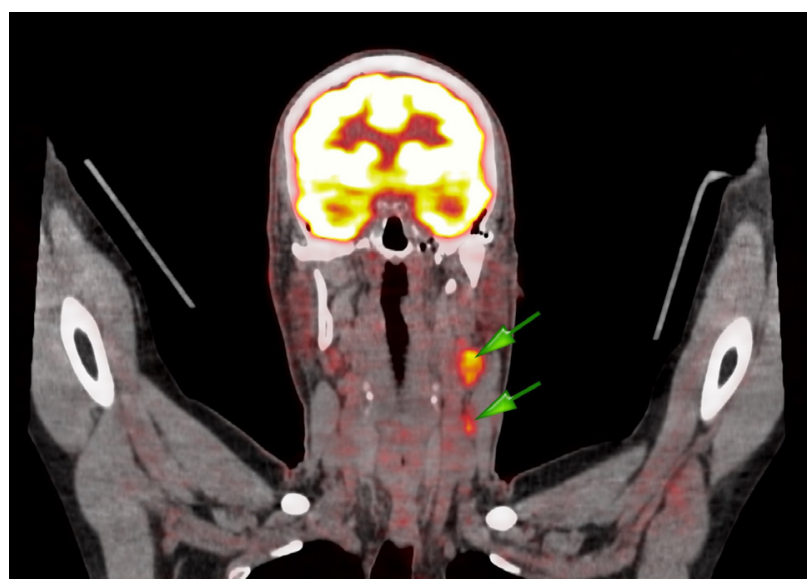

Figure 2. PET with ${ }^{18} F-F D G$ showed active metabolic process in several lymph nodes of the upper and middle part of the neck (green arrows) 


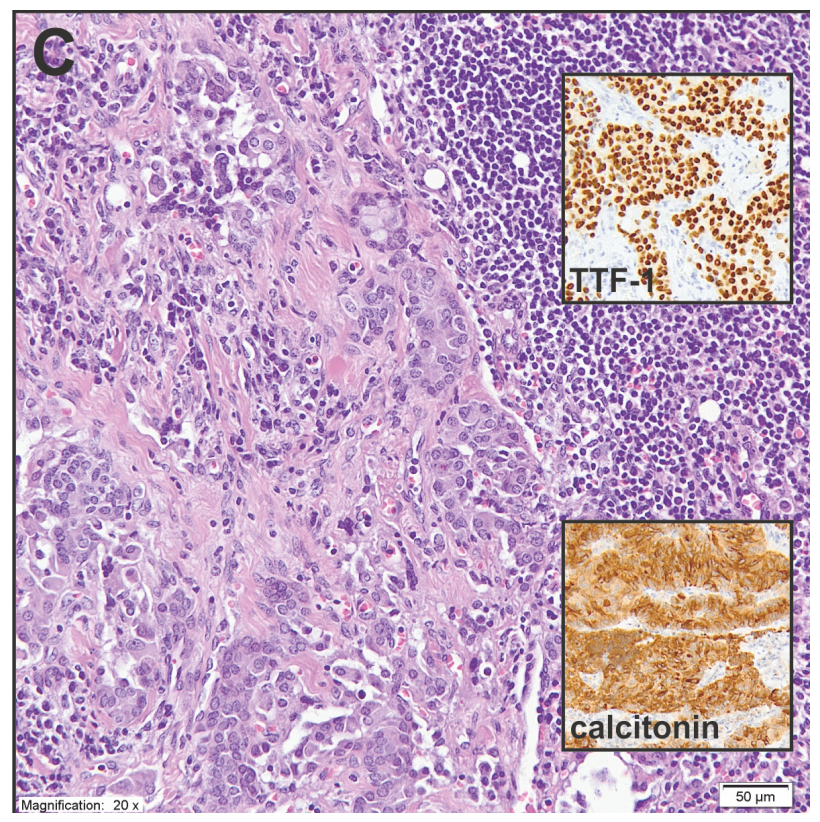

Figure 3. Histological images of both neoplasms. Nodal metastasis of the medullary carcinoma composed of cells with grainy (salt and pepper) chromatin showing TTF-1 and calcitonin expression (upper and lower inlet, respectively)

patients for a radical thyroidectomy with radical neck dissection. The resected specimens were evaluated by two experienced histopathologists, which resulted in identification of multifocal papillary thyroid carcinoma (PTC) in the thyroid gland (pT1a[m]N0) and positive MTC metastases in central and left-sided LNs (levels II-IV). Congruent to MRI and PET scan, histopathological examination also failed to determine the site of primary MTC tumour (pTxN1b) (Fig. 3, 4).

The postoperative period was uneventful. MEN2 syndrome was excluded on the basis of negative MRI for pheochromocytoma and negative genetic tests for RET and BRAF mutations. On one-year follow-up, the patient was doing well with serum calcitonin level below $2 \mathrm{pg} / \mathrm{mL}$.

Simultaneous medullary (MTC) and papillary thyroid carcinoma is extremely rare [1]. To our knowledge, such coexistence without the identification of a primary tumour site for either carcinoma has never been reported. In our case, the primary origin of MTC was not identified even after extensive imaging tests and histopathological examination of thyroidectomy specimen (by two experienced pathologists). When a diligent search fails to disclose the primary lesion, then the metastatic disease is classified as "carcinoma of unknown primary" syndrome [2]. This may have occurred in our case, because the primary MTC was small in size, induced metastasis, and then regressed completely while metastatic lesion proliferated in the LNs [3].

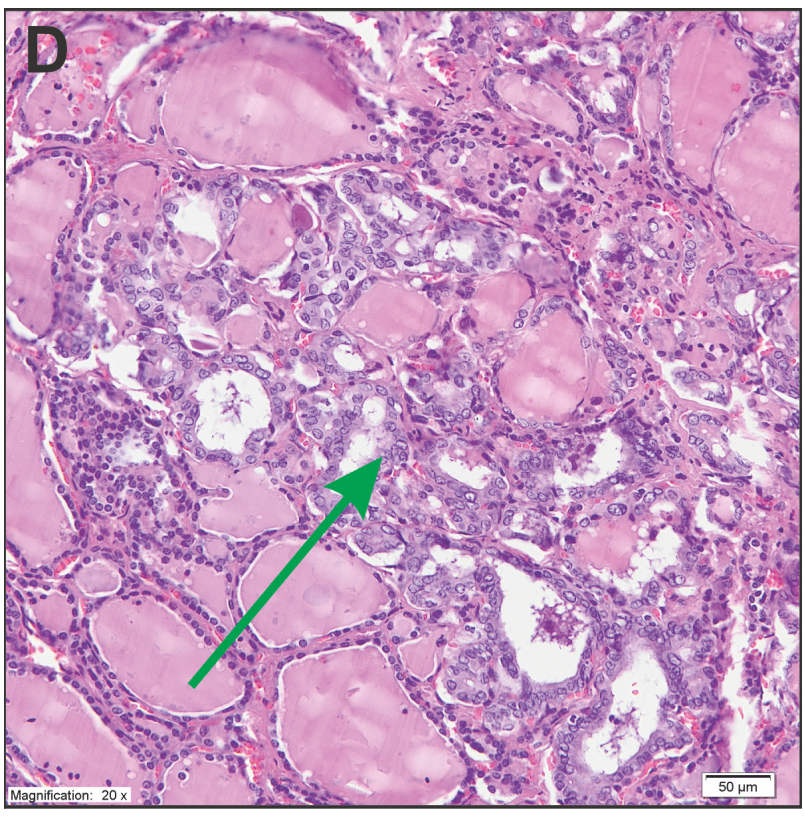

Figure 4. A small focus of papillary carcinoma in the thyroid gland (green arrow)

\section{Acknowledgement}

None.

\section{Statement of ethics}

Written, informed consent was given by the patient for publishing this case.

\section{Disclosure statement}

The authors have no conflicts of interest to declare.

\section{Funding sources \\ None}

\section{Author contributions}

A.R.H. and A.P. were involved in the conception of the manuscript, drafting the manuscript, and approval of the final version. M.S., L.O., M.B., B.M., and L.K. were involved in the conception of the manuscript and approval of the final version. A.R.H., M.S., L.O., M.B., B.M., and L.K. were involved in the treatment of the patient.

\section{References}

1. Machens A, Dralle H. Simultaneous medullary and papillary thyroid cancer: a novel entity? Ann Surg Oncol. 2012; 19(1): 37-44, doi: 10.1245/s10434-011-1795-z, indexed in Pubmed: 21626080.

2. Raber MN. Carcinoma of unknown primary origin: on the cusp of a new era. Cancer J. 2010; 16(1): 66-67, doi: 10.1097/PPO.0b013e3181ce4769, indexed in Pubmed: 20164693.

3. Conway AM, Mitchell C, Kilgour E, et al. Molecular characterisation and liquid biomarkers in Carcinoma of Unknown Primary (CUP): taking the 〈U〉 out of 〈CUP〉. Br J Cancer. 2019; 120(2): 141-153, doi: 10.1038/s41416-018-0332-2, indexed in Pubmed: 30580378. 